\title{
Implementation of DSP System for Discrete Transforms using VHDL
}

\author{
Deepak Kumar ${ }^{1}$ \\ M.Tech. (Scholar) \\ Dept.of Electronics Engineering, \\ Pondicherry University, Puducherry ${ }^{1}$
}

\author{
K.Anusudha ${ }^{2}$ \\ Assistant Professor, \\ Dept. of Electronics Engineering, \\ Pondicherry University, Puducherry. ${ }^{2}$
}

\begin{abstract}
Discrete Cosine transform (DCT), Inverse DCT, Discrete Fourier Transform (DFT) and Fast Fourier Transform (FFT) are performed by DSP system on one platform. This paper represent the design of a Digital Signal Processor (DSP) system described using VHDL and implement in a Field Programmable Logic Array (FPGA). The Fast Fourier Transform (FFT) is one of the rudimentary operations in field of digital signal and image processing. Some of the very vital applications of the fast Fourier transform include Signal analysis, Sound filtering, Data compression, Partial differential equations, Multiplication of large integers, Image filtering etc. Fast Fourier transform (FFT) is an efficient implementation of the Discrete Fourier Transform (DFT). This paper concentrates on the development of the Fast Fourier Transform (FFT), based on Decimation-In-Time (DIT) domain, Radix-2 algorithm, this paper uses VHDL as a design entity, and their Synthesis by Xilinx Synthesis Tool. The input of Fast Fourier transform has been given by testbench and output has been displayed using the waveforms on the Xilinx Design Suite 13.1.The synthesis results show that the computations for calculating the Fast Fourier transform. By using a fixed geometry addressing, pipeline designing and block fixed point structure, the data has the greater precision and dynamic range. The results show that the design is efficient, strongly extensive and occupies less resource. It is a good method to meet the high-speed digital signal processing requirements.
\end{abstract}

\section{General Terms}

Algorithm, embedded, simulation, performance.

\section{Keywords}

Control Unit (CU), General Purpose Register (GPR), Register Set (RS), Multiply and accumulates (MACs), Very Large Instruction Word (VLIW).

\section{INTRODUCTION}

A digital signal processor is a specialized microprocessor with an architecture optimized for the fast operational needs of digital signal processing. Digital signal processing algorithms typically require a large number of mathematical operations to be performed quickly and repeatedly on a set of data. Signals are constantly converted from analog to digital, manipulated digitally, and then converted back to analog form. Many DSP applications have constraints on latency; that is, for the system to work, the DSP operation must be completed within some fixed time, and deferred processing is not viable.

Most general-purpose microprocessors and operating systems can execute DSP algorithms successfully, but are not suitable for use in portable devices such as mobile phones and
PDAs because of power supply and space constraints. A specialized digital signal processor, however, will tend to provide a lower-cost solution, with better performance, lower latency, and no requirements for specialized cooling or large batteries.

Main features of DSP are:

- Special Single Instruction Multiple Data (SIMD) operations

- Perform DCT (Discrete Cosine transform) and IDCT (Inverse Discrete Cosine transform).

- Perform DFT (Discrete Fourier Transform) and FFT (Fast Fourier Transform).

- It can be implemented in general purpose computers or with embedded processors that may or may not include specialized microprocessors called digital signal processors.

- Use VLIW (Very Large Instruction Word) techniques so each instruction drives multiple arithmetic units in parallel.

- Special arithmetic operations, such as fast Multiply and Accumulates (MACs).

- Many fundamental DSP algorithms, such as FIR filters or the Fast Fourier transform depend heavily on multiply-accumulate performance.

- Bit-reversed addressing, a special addressing mode useful for calculating FFTs.[2]

Digital Signal Processor is optimized specially for digital signal processing. It also support features as an applications processor or microcontroller. DSP operations process the continuous signals and data. A Digital Signal Processor is a specialized microprocessor with an architecture developed for the fast operational needs of digital signal processing.

A digital signal processor (DSP) is a specialized microprocessor with an architecture optimized for the fast operational needs of digital signal processing. Digital signal processing algorithms typically require a large number of mathematical operations to be performed quickly and repeatedly on a set of data. Signals (perhaps from audio or video sensors) are constantly converted from analog to digital, manipulated digitally, and then converted back to analog form. Many DSP applications have constraints on latency; that is, for the system to work, the DSP operation must be completed within some fixed time, and deferred (or batch) processing is not viable Most general-purpose microprocessors and operating systems can execute DSP algorithms successfully, but are not suitable for use in portable devices such as mobile phones and PDAs because of power supply and space constraints. A specialized digital signal processor, however, will tend to provide a lower-cost solution, with better performance, lower latency, and no 
requirements for specialized cooling or large batteries. A digital signal processor (DSP) is a specialized microprocessor with an architecture optimized for the fast operational needs of digital signal processing.

1.1.1 The paper is organized in such a way that the section 2 briefs the proposed DSP Architecture, section 3 explains the instruction format, section 4 deals with the instruction set; section 5 shows DSP operations, section 6 gives the simulation results for different inputs. Finally, section 7 summaries the paper.

\section{DSP ARCHITECHTURE}

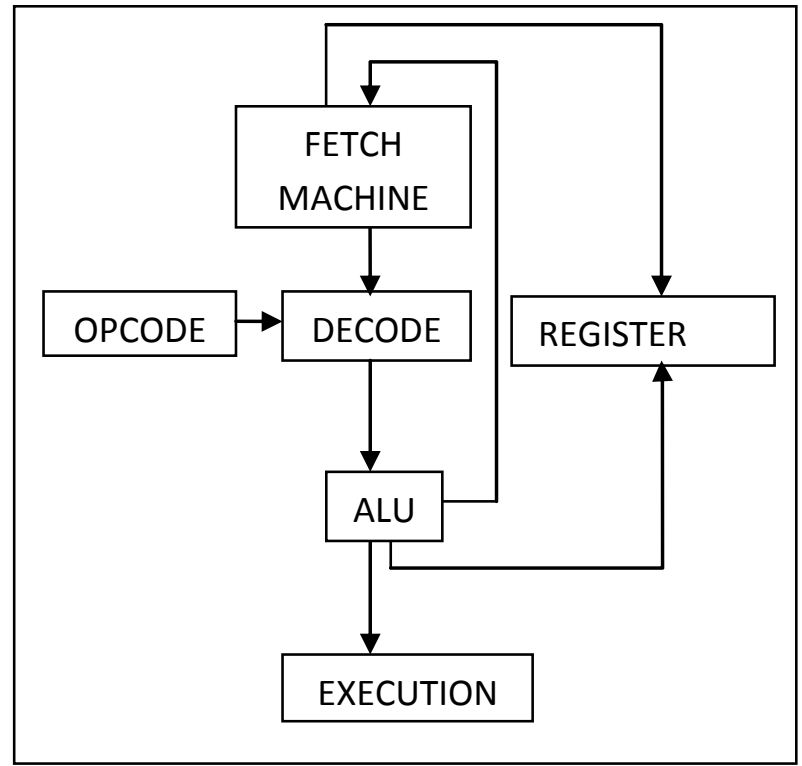

Fig 1: DSP System Architecture

Register Set (RS): In this information is encoded, stored, and retrieved. Up to 64 GPRs can be used in this architecture. All GPRs can be used in any operation except for the load and store instructions. Only GPR can be used for loading and storing.

Fetch Machine: This machine fetches an instruction from external memory, and upon completion of the instruction fetch cycle this machine signals the decoder to decode the instruction. This machine utilizes a 3-bit up-counter with an active low reset. The CPU changes states and begins to decode the instruction.

Decoder: Upon completion of the instruction fetch cycle, the instruction is decoded. The decoder reads bit 3 down to 0 of the IR, decides which of the sixteen operations the CPU needs to performs, and signals one of the next states to begin its operation.

Move Machine: The move machine controls all register movement. The most basic of these movements is the movement of data from one GPR to another GPR.Upon completion of the movement of data, a new instruction is fetched.

Arithmetic Logic Unit: The ALU performs arithmetic and logical operations on data. The data is taken from two GPRs and is moved to the ALU. The result is stored in a GPR. For operations that involve one operand, a GPR can be specified to store the result. The ALU supports two's complement data.

Execution: after performing operation execution machine giving output and result will show in output device.

\section{INSTRUCTION FORMAT}

The DSP is 20-bit processor so that the value of each instruction is 20 bits. Each instruction decodes by internal decoder and DSP machine fetches an instruction from the memory. In this DSP two 8-bit instructions are coming as inputs i.e. 8-bit $\mathrm{x}$ or 8-bit $\mathrm{y}$. and 0 to 3 bits is the opcode for instruction of performed operations.

Table no.1 shows the input instruction format. [5]

TABLE I

INSTRUCTION FORMAT for INPUT

\begin{tabular}{|c|c|c|c|c|}
\hline \multicolumn{2}{|c|}{$\mathrm{R}[\mathrm{y}]$} & \multicolumn{2}{|c|}{$\mathrm{R}[\mathrm{x}]$} & OPCODE \\
\hline 19 & 12 & 11 & 4 & 0 \\
\hline
\end{tabular}

\section{INSTRUCTION SETS}

The processor with 4 bit opcode to allow instruction to perform various operations such as or ,and, nand, nor , xor, xnor , add , subtract, not , increment, decrement, etc. Each opcode has specific operation in processor; RISC have 4bit opcode that can perform 16 different operations. Table 2 shows the instruction set for RISC processor.

TABLE II

INSTRUCTION SETS

\begin{tabular}{c|l|l|} 
OPCODE & FUNCTION & Operation performed \\
$\mathbf{0 0 0 0}$ & DCT & DCT operation of one register \\
$\mathbf{0 0 0 1}$ & IDCT & IDCT operation of one register \\
$\mathbf{0 0 1 0}$ & FFT & FFT operation of one register \\
$\mathbf{0 0 1 1}$ & IFFT & IFFT operation of one register
\end{tabular}

\section{DSP OPERATIONS}

\subsection{Discrete Fourier Transform}

It is a kind of Discrete Transform which is used in Fourier analysis. It transforms one function into another, which is called the frequency domain representation, or simply the DFT, of the original function.

- DFT is used in Fourier analysis.

- It is Frequency domain representation of signal.

The formula for DFT is. [3]

$$
X(k)=\sum_{n=0}^{N-1} x[n] e^{-j \frac{2 \pi}{N} n k} \quad \begin{array}{r}
k=0,1, \ldots N-1 \\
x(n T)=x[n]
\end{array}
$$

The N-point DFT of time samples is $\mathrm{x}(0), \mathrm{x}(1), \mathrm{x}(3), \ldots, \mathrm{x}(\mathrm{N}-1)$ defined as

$$
X[n]=\sum_{m=0}^{N-1} x[m] e^{-j 2 \pi m n / N}=\sum_{m=0}^{N-1} x[m] w_{N}^{m n} \quad(n=0,1, \cdots, N-1)
$$

Let $\mathrm{N}=2 \mathrm{M}$ the DFT can be written as

$$
X[n]=\sum_{m=0}^{N-1} x[m] w_{N}^{m n}=\sum_{m=0}^{M-1} x[2 m] w_{2 M}^{2 m n}+\sum_{m=0}^{M-1} x[2 m+1] w_{2 M}^{(2 m+1) n}
$$




$$
\begin{gathered}
w_{2 N}^{2 k}=e^{-j 2 \pi k 2 / 2 N}=e^{-j k 2 \pi / N} \equiv w_{N}^{k} \\
w_{2 N}^{N}=e^{-j 2 \pi N / 2 N}=e^{-j \pi} \equiv-1
\end{gathered}
$$

\subsection{Fast Fourier Transform-}

FFT is an efficient algorithm or fast way to compute a DFT. Radix-2 Decimation-in-time (DIT) Fast Fourier Transform (FFT) is dividing the DFT in to two portions. The Basic Butterfly [1] operation of radix-2 DIT FFT algorithm of 8 signals is shown in Fig2.[3]

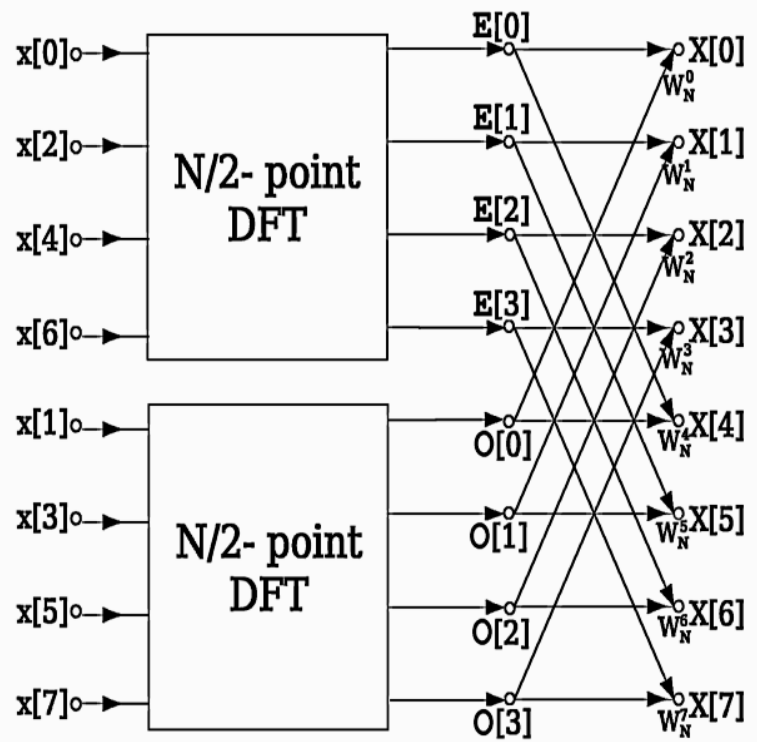

Fig 2: Butterfly structure

\subsection{DCT \& IDCT -}

A discrete cosine transform (DCT) expresses a sequence of finitely many data points in terms of a sum of cosine functions oscillating at different frequencies.

The N-point 1-D IDFT is defined as:

$$
X[n]=\sum_{m=0}^{N-1} x[m] c[n, m] \quad(n=0, \cdots, N-1)
$$

where $c[m, n]$ is modified with $a[n]$ which is also the component in the $\mathrm{n}^{\text {th }}$ row and $\mathrm{m}^{\text {th }}$ column of the $\mathrm{N}$ by $\mathrm{N}$ cosine transform matrix.

$$
\left[\begin{array}{ccc}
\cdots & \cdots & \cdots \\
\cdots & c[n, m] & \cdots \\
\cdots & \cdots & \cdots
\end{array}\right]=\left[\begin{array}{c}
\mathbf{c}_{0}^{T} \\
\cdots \\
\mathbf{c}_{N-1}^{T}
\end{array}\right]=\mathbf{C}^{T}
$$

Now the DCT can be expressed in matrix form as:

$$
X[n]=\sum_{m=0}^{N-1} x(m) * C^{\wedge} t \text { Or } \quad \mathbf{X}=\mathbf{C}^{T} \mathbf{x}
$$

Sequence of finite many data points in terms of a sum of cosine functions oscillating at different frequencies.

The basic operation of the DCT is as follows:

- The input image is $\mathrm{N}$ by $\mathrm{M}$;

- $f(i, j)$ is the intensity of the pixel in row $i$ and column $j$;

- $\mathrm{F}(\mathrm{u}, \mathrm{v})$ is the DCT coefficient in row $\mathrm{k} 1$ and column $\mathrm{k} 2$ of the DCT matrix.

- For most images, much of the signal energy lies at low frequencies; these appear in the upper left corner of the DCT.

- Compression is achieved since the lower right values represent higher frequencies, and are often small - small enough to be neglected with little visible distortion.

- The DCT input is an 8 by 8 array of integers. This array contains each pixel's gray scale level;

- 8 bit pixels have levels from 0 to 255. [2][6]

\section{SIMULATION RESULTS}

This design is synthesized in Xilinx 13.1 ISE web pack. The simulation is done by integrated testbench in 13.1 version. All the operation can be simulated at one time. The behavioral simulation is done by executing the test bench file. The testing is done by checking the syntax of the code or by checking the sentences of the code.After completed the coding of basic architecture of RAM \& DSP we have to write the coding for test bench to call the codes of DSP to execute the codes.

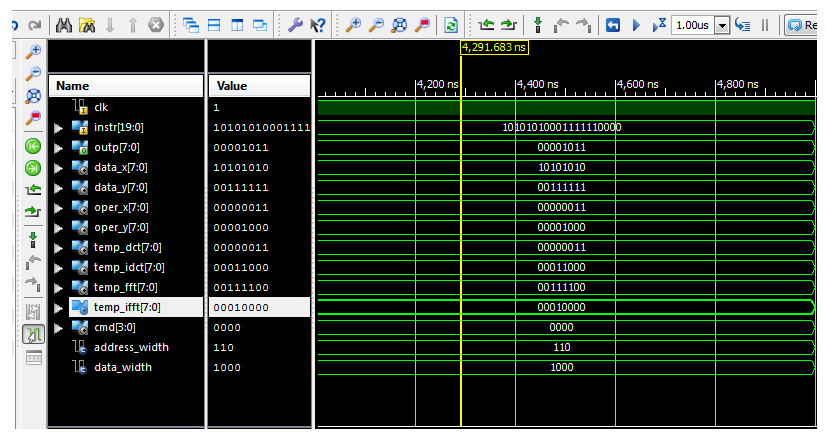

Fig 3: Simulation result.
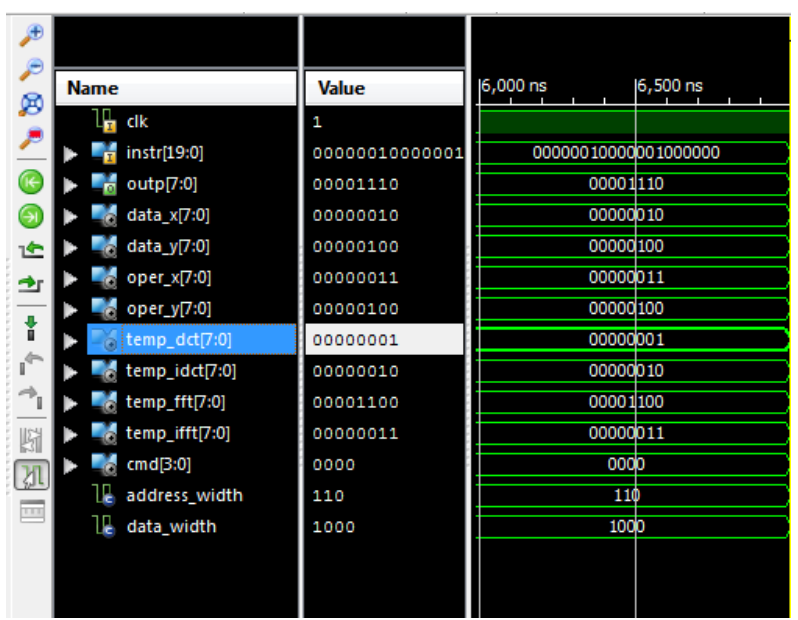

Fig 4: Simulate Behavioral Model.

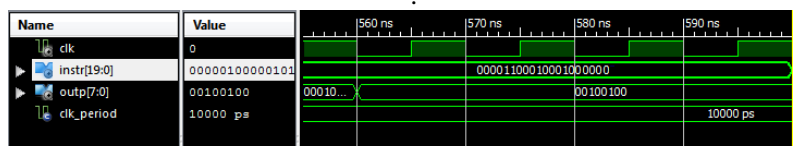


Fig5: output for DCT operation.

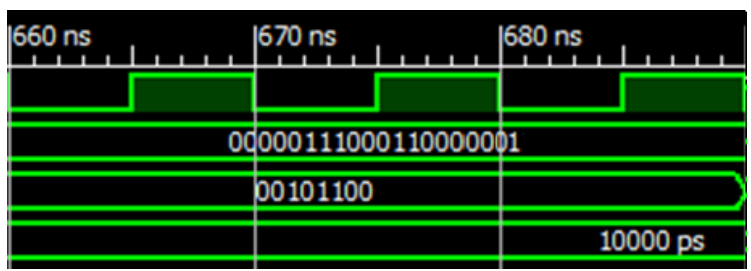

Fig 6: output for IDCT operation.

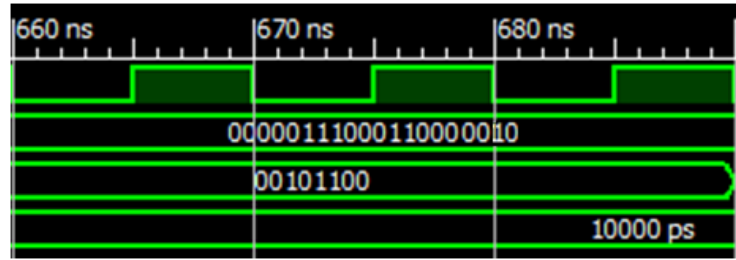

Fig 7: output for FFT operation.

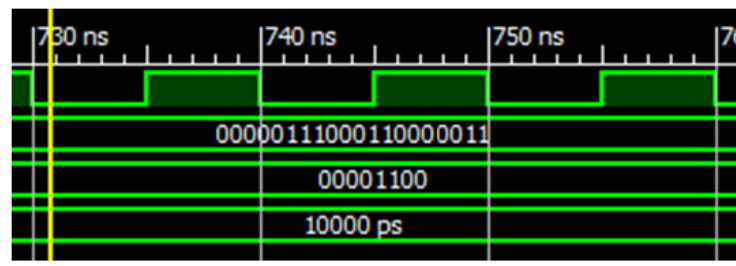

Fig 8: output for IFFT operation.

Input $\mathrm{y}=$ "01000100", Value store in 'y' reg. $=05, \mathrm{Y}=$ $[5,5,5,5]$ Operation $=\mathrm{C}^{\mathrm{T}} \mathrm{y}$, (Calling library function for DCT operation $), \mathrm{o} / \mathrm{p}=\left(\mathrm{y}_{1}+\mathrm{y}_{2}+\mathrm{y}_{3}+\mathrm{y}_{4}\right), \mathrm{o} / \mathrm{p} \mathrm{DCT}=36=00100100$; The input for IDCT is 00000111 at 0001 opcode and output is 00101100 , the input for FFT operation is 00000111 at 0010 opcode and output is 00101100; input for IFFT operation is 00000111 at 0011 opcode and output is 00001100 .

\section{CONCLUSION}

The simulation results of this processor provide various features including arithmetic operations and Fourier transform. Discrete Cosine transform (DCT), Inverse DCT, Discrete Fourier Transform (DFT) and Fast Fourier Transform (FFT) can be performed by the proposed DSP system using VHDL and synthesized in Xilinx 13.1 ISE web pack. The design implemented can be easily seen in the waveform. This design can be easily upgraded by increasing the memory of the processor and can be implemented with higher bit value. The design has been achieved using VHDL. Simulation results show that the processor is working perfectly and executes all the instructions in one clock cycle.

\section{REFERENCES}

[1] Asmita Haveliya "Design and Simulation of 32-Point FFT Using Radix-2 Algorithm for FPGA Implementation" IEEE $2^{\text {nd }}$ International Conference on Advanced Computing \& Communication Technologies, pp. 167-171, Jan. 2012, India.

[2] S. Belkouch, M. El Aakif and A. Ait Ouahman "Improved Implementation of a Modified Discrete Cosine Transform on Low-Cost FPGA" IEEE 5th International Symposium on $\mathrm{I} / \mathrm{V}$ Communications and Mobile Network, Oct 2010, Rabat, Morocco.
[3] Zi-Wei Zheng and Zhe Ren "Efficient Design of Fast Fourior Transform Processor Using FPGA Technology" IEEE International Conference on Electrical and Control Engineering, pp.5195-5198, June 2010, Wuhan, China.

[4] LI Xiao-feng, Chen Long, Wang Shihu "The Implementation of High-speed FFT processor based on FPGA" IEEE International Conference on Computer, Mechatronics, Control \& Electronic Engineering (CMCE), Vol.2 pp. 236-239, Aug 2010, Changchun, China.

[5] Jarrod D. Luker and Vinod B. Prasad "RISC System Design in an FPGA" IEEE Conference Publication, Vol. 2, pp. 532-536, Aug 2001, Dayton, USA.

[6] Atitallah, A.B.; Kadionik, P.; Ghozzi, F.; Nouel, P.; Masmoudi, N.; Marchegay, P., "Optimization and Implementation on Fpga of the DCT/IDCT Algorithm" IEEE International Conference on Acoustics, Speech and Signal Processing, vol. 3 pp. May 2006.Toulouse, France.

[7] Giovanni Betta, Senior Member, IEEE, Consolatina Liguori, Member, IEEE, and Antonio Pietrosanto, Member, IEEE, "A Multi-Application FFT Analyzer Based on a DSP Architecture", IEEE International Transactions on Instrumentation And Measurement, vol. 50, Issue: 3, pp. 825 - 832, Jun 2001.

[8] S. Akrouf, M. A. Sehili, A. Chakhchoukh, M. Mostefai and C. Youssef, "Face recognition using PCA and DCT," $5^{\text {th }}$ International Conference on MEMS, NANO, and smart systems (ICMENS), , pp. 15-19, 2009.

[9] Brown, Richard B.; Lomax, Ronald J.; Carichner, Gordon A.; Drake, Alan J. "A microprocessor design project in an introductory VLSI course" IEEE Trans. Educ., vol. 43, pp. 353 - 361, Aug 2000.

[10] John L. Hennessy, and David A. Patterson, 2006. "Computer Architecture A Quantitative Approach",

[11] Leon W. Couch, 1997. Digital and Analog Communication Systems, Prentice Hall. New Jersey.

[12] Don Anderson, 1997. Universal Serial Bus System Architecture. Addison Wesley. Boston.

\section{AUTHORS' PROFILES}

Deepak Kumar received the B.E. degree in Electronics and Communication Engineering from the Rajeev Gandhi Prodyogiki Visvavidhyalaya Bhopal, Madhya Pradesh, India in 2010. Currently, he is studying M. Tech (Electronics) in the Dept. of Electronics Engineering, School of Engineering and Technology, Pondicherry University, Puducherry, India.

K.Anusudha received the B.E degree (2002) in Electronics \&Communication from Madras University, M. Tech degree (2004) in communication system from Anna University, India. She is currently pursuing $\mathrm{PhD}$. She is currently working as Assistant Professor in the Dept. of Electronics Engineering, School of Engineering and Technology, Pondicherry University, Puducherry, India. Her research interests include Digital data security, Digital watermarking and Forensic informatics. 